TITLE:

\title{
Direct determination of Burgers vector sense and magnitude of elementary dislocations by synchrotron white x-ray topography
}

\section{$\operatorname{AUTHOR}(S)$ :}

Nakamura, Daisuke; Yamaguchi, Satoshi; Hirose, Yoshiharu; Tani, Toshihiko; Takatori, Kazumasa; Kajiwara, Kentarou; Kimoto, Tsunenobu

\section{CITATION:}

Nakamura, Daisuke ...[et al]. Direct determination of Burgers vector sense and magnitude of elementary dislocations by synchrotron white $x$-ray topography. JOURNAL OF APPLIED PHYSICS 2008, 103(1): 013510.

\section{ISSUE DATE:}

2008-01-01

URL:

http://hdl.handle.net/2433/84577

\section{RIGHT:}

Copyright 2008 American Institute of Physics. This article may be downloaded for personal use only. Any other use requires prior permission of the author and the American Institute of Physics. 


\title{
Direct determination of Burgers vector sense and magnitude of elementary dislocations by synchrotron white x-ray topography
}

\author{
Daisuke Nakamura ${ }^{\text {a) }}$ \\ Toyota Central Research and Development Laboratories Incorporated, Nagakute-cho, Aichi 480-1192, \\ Japan and Department of Electronic Science and Engineering, Kyoto University, Katsura, Nishikyo, \\ Kyoto 615-8510, Japan \\ Satoshi Yamaguchi, Yoshiharu Hirose, Toshihiko Tani, and Kazumasa Takatori \\ Toyota Central Research and Development Laboratories Incorporated, Nagakute-cho, Aichi 480-1192, \\ Japan \\ Kentarou Kajiwara \\ Japan Synchrotron Radiation Research Institute (JASRI), SPring-8, Sayo-cho, Hyogo, 679-5198, Japan \\ Tsunenobu Kimoto \\ Department of Electronic Science and Engineering, Kyoto University, Katsura, Nishikyo, Kyoto 615-8510, \\ Japan
}

(Received 22 August 2007; accepted 7 November 2007; published online 14 January 2008)

\begin{abstract}
The x-ray topography by using highly coherent beam obtained at third-generation synchrotron facilities can provide higher spatial resolution and higher lattice-distortion sensitivity than those by former-generation facilities. Here, we report the direct determination of the Burgers vector senses and magnitudes of elementary dislocations in a high-quality silicon carbide single crystal using white $\mathrm{x}$-ray section topography with a long sample-to-film distance. Our data strongly indicate that there are very weak but extraordinarily long-range elastic interactions between elementary screw dislocations. Those interactions govern dislocation-propagation behavior and the distribution of dislocations. Moreover, we found that white x-ray projection topography with a long sample-to-film distance can also be a powerful tool to effectively examine the detailed structure of elementary dislocations in single crystals. (C) 2008 American Institute of Physics. [DOI: 10.1063/1.2829806]
\end{abstract}

\section{INTRODUCTION}

Silicon carbide $(\mathrm{SiC})$ is a promising material for nextgeneration high power devices. ${ }^{1-3}$ The performance and reliability of $\mathrm{SiC}$ devices, however, is seriously impaired by the high density of crystalline defects in single crystal wafer, a problem which limits the range of their application. Although the most harmful defect of a micropipe (hollow-core superscrew dislocation) has been eliminated from recent commercial wafers, ${ }^{4,5}$ a high density of elementary dislocations (threading dislocation, basal-plane dislocation, and partial dislocation) still exist in the wafers. These elementary dislocations are also thought to be harmful to device performance and especially to long-term reliability of the devices. ${ }^{6,7}$ Still, it is not clear which of the elementary dislocations interfere with the device reliability, probably because we lack a fundamental understanding of the properties of the dislocations and their structure.

The authors have reported the use of novel growth process, repeated a-face (RAF) growth process, to reduce the density of these elementary dislocations in $\mathrm{SiC}$ crystals by two orders of magnitude (compared to that of conventional crystals).$^{8}$ Moreover, unique types and structure of the elementary dislocations in the crystals with low dislocation density have been revealed using bulk-sensitive $\mathrm{x}$-ray topography. ${ }^{9}$ As we showed in our previous study, most of

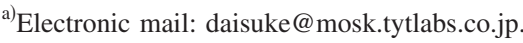

long-threading (LT) dislocations in RAF-grown $4 H$-SiC crystals are not the well-known pure screw dislocations with a Burgers vector of $1 c(\langle 0001\rangle)$ but mixed dislocations with a Burgers vector of both $c$ and $a(1 / 3\langle 11 \overline{2} 0\rangle)$. In fact, whether the LT dislocations are pure screw or mixed, they have relatively large Burger vector magnitude of approximately $1 \mathrm{~nm}$ or more. This large magnitude of Burgers vector suggests that there is relatively large distortion around the screw dislocations (here, we refer to the LT dislocation as a screw dislocation in accordance with customary expression) and might result in a relatively serious impairment of device reliability. These facts suggest that the fundamental properties of screw dislocations are very important both from the viewpoint of understanding device properties and crystal growth.

So far, the Burgers vector sense and magnitude of dislocation (some of important properties of dislocation, along with the Burger vector direction, and dislocation-line direction) have not been sufficiently discussed in the context of device properties and crystal growth, probably because it is difficult to determine both the Burgers vector sense and magnitude of the elementary dislocation by means of conventional dislocation-analysis techniques such as chemical etching, polarized light microscopy, and classical x-ray topography. Although there were a few reports on the determination of the Burgers vector sense and magnitude of the elementary dislocation by the x-ray topography techniques, ${ }^{10}$ very recently, some excellent works using grazing-incidence synchrotron white $\mathrm{x}$-ray topography ${ }^{11,12}$ have been reported 


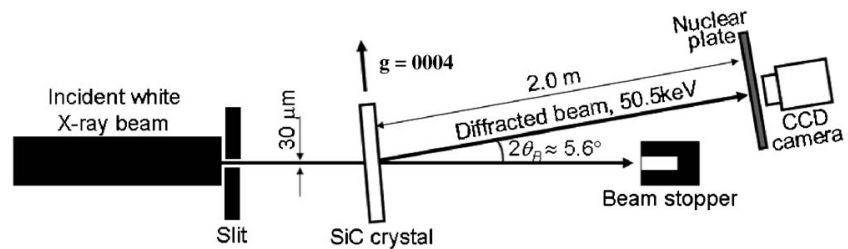

FIG. 1. Schematic of experimental setup for section topography. Stripeshaped beam was obtained by limiting the height of incident white x-ray with a slit. The stripe-shaped white $\mathrm{x}$-ray is diffracted by high-quality $4 H$-SiC with a diffraction vector $\mathbf{g}=0004$. Owing to white $\mathrm{x}$-ray, the Bragg condition can be satisfied even on the lattice plane distorted by a screw dislocation. The redshifted and blueshifted beam diffracted on the distorted lattice plane near the screw dislocation deviates with a minute angle compared to that far from the dislocation. The small angular dispersion of the diffracted beam can be detected on a film (nuclear plate) placed at a distance of $2.0 \mathrm{~m}$ from the sample.

by Chen et al. so that the detailed discussion including the Burgers vector sense and magnitude of the elementary dislocations could be explored.

In this study, we try to determine the Burgers vector sense and magnitude of the screw dislocation by means of white $\mathrm{x}$-ray section/projection topography with a long sample-to-film distance. Owing to our use of the RAF-grown high-quality sample and a highly coherent third-generation synchrotron beam, we have been able to observe the lattice distortion near the dislocation with high signal-to-noise ratio and high lattice-distortion sensitivity. Based on these results, we have been able to shed new light on dislocationpropagation behavior during crystal growth.

\section{EXPERIMENT}

The growth technique we used to prepare our highquality $\mathrm{SiC}$ single crystal is a well-known sublimation technique. ${ }^{13}$ Growth temperature and growth rate were about $2200-2400{ }^{\circ} \mathrm{C}$ and $0.5-1.0 \mathrm{~mm} / \mathrm{h}$, respectively. RAFgrown ingots were manufactured using a process consisting of three steps of growth along $[11 \overline{2} 0] \Rightarrow[1 \overline{1} 00] \Rightarrow[000 \overline{1}]$. The final $[000 \overline{1}]$-grown ingot was cut in the direction of its growth, and an in-house RAF $4 H-S i C$ substrate was prepared as a sample. The surface orientation of the sample was perpendicular to both the directions of its growth and $\langle 0001\rangle$. The dimension of the sample was $50 \times 10 \times 0.40 \mathrm{~mm}$. The sample surface was carefully polished and treated to eliminate mechanically damaged layer on both sides so that it could be examined by transmission x-ray topography. The screw dislocation density of this sample is estimated to be about $500 \mathrm{~cm}^{-2}$. This value is lower by one order of magnitude than that used in previous works. ${ }^{14-19}$

We carried out our observations of synchrotron white $\mathrm{x}$-ray beam topography at SPring-8, the second hutch of BL28B2. We took both white $\mathrm{x}$-ray section and projection topographic images for comparison. The optical geometry of the white $\mathrm{x}$-ray section topography is shown in Fig. 1. The height of incident white $\mathrm{x}$-ray beam was limited to about $30 \mu \mathrm{m}$ by a slit. All the topographs were taken in symmetric transmission with a diffraction vector $\mathbf{g}=0004$. The specimen-to-film distance was $2.0 \mathrm{~m}$, with the expectation that the spatial resolution of our topographs would be ap-
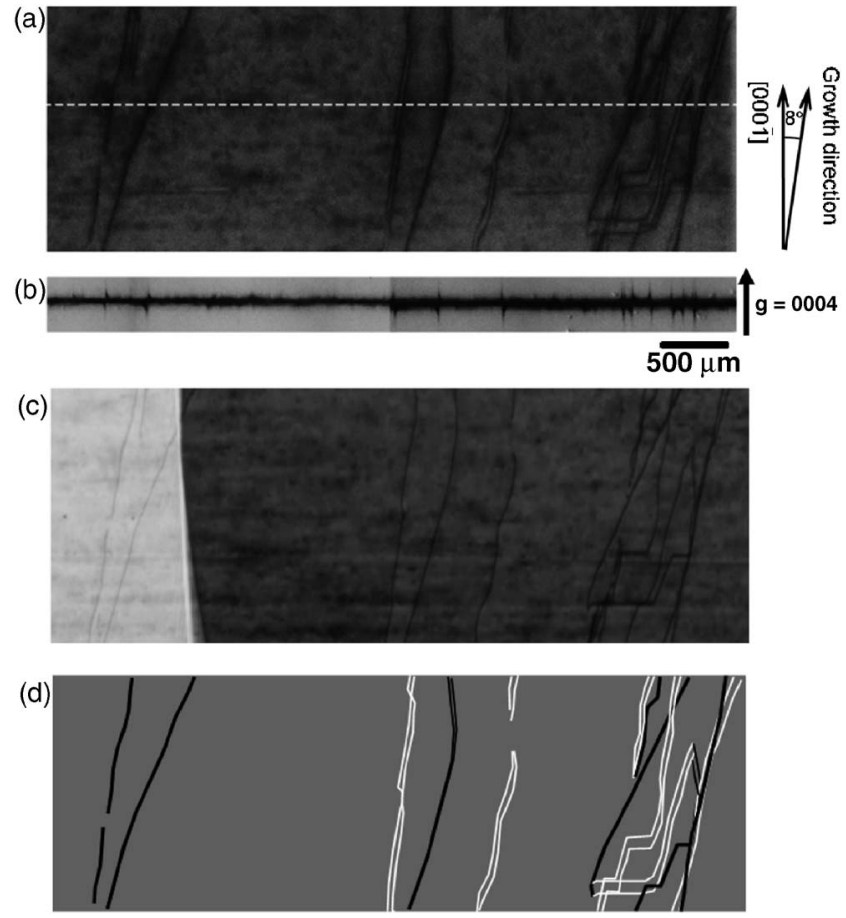

FIG. 2. Synchrotron white $\mathrm{x}$-ray topographs, projection topograph (a) and section topograph (b) obtained at the broken line of (a). (c) is a synchrotron monochromatic x-ray topograph taken at the same area as (a) (white area on the left edge is due to shading diffracted beam by a sample holder). (d) is a simplified schematic of (a), in which right-handed and left-handed dislocations are indicated by black and white, respectively.

proximately $7 \mu \mathrm{m}$ horizontally and $1 \mu \mathrm{m}$ vertically (assuming a light source of $150 \times 10 \mu \mathrm{m}^{2}$ in a rectangular size, with a source-to-sample distance of $44 \mathrm{~m}$ ). The optical geometry of the white $\mathrm{x}$-ray projection topography was almost the

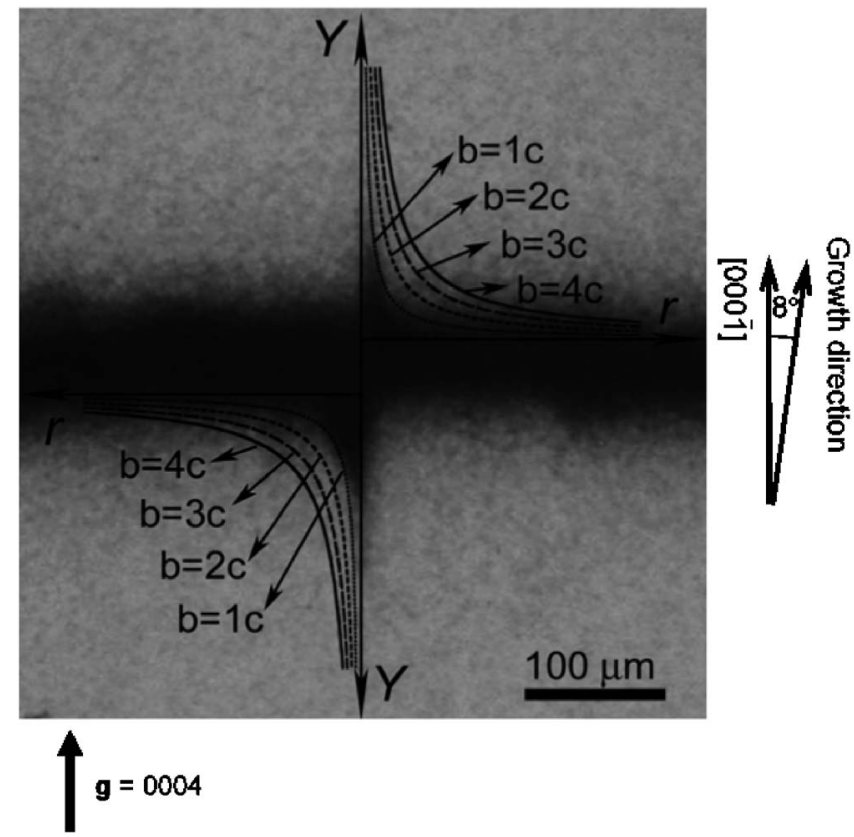

FIG. 3. Magnified image of section topograph of Fig. 2(b). The screw dislocation that originates from this hyperbolic profile is right handed. Theoretical hyperbolic curves for the Burgers vectors of $1 c, 2 c, 3 c$, and $4 c$ are also drawn on the observed section topograph. It is clear that the curve for $b=1 c$ best fits the experimental profile. 
same as the section topography except for the beam height of $5 \mathrm{~mm}$. All the topographs were recorded on nuclear plates, Ilford L4, with a high resolution of several microns.

\section{RESULTS}

Figures 2(a) and 2(b) show the white $\mathrm{x}$-ray projection topograph and white $\mathrm{x}$-ray section topograph, respectively. A monochromatic $\mathrm{x}$-ray projection topograph taken at the same area as Fig. 2(a) is also shown in Fig. 2(c) for comparison. The section topograph of Fig. 2(b) was obtained at the position of white broken line in Fig. 2(a). Since both topographs were taken in symmetric transmission with diffraction vector $\mathbf{g}=0004$, dislocation images in Fig. 2 should be primarily due to the Burgers vector component parallel to $\langle 0001\rangle$ ( $c$ axis). The screw dislocations with the Burgers vector component parallel to $c$ axis appear as dark lines in Fig. 2(a), which tend to lie along the growth direction (not exactly along the $c$ axis). Their dislocation lines, however, are neither perfectly straight nor perfectly parallel to the growth direction. Most of them wind slightly, and some of them lie partially in the (0001) plane. These slightly winding dislocation lines in the present crystal might be a peculiar characteristic of the screw dislocations in $4 \mathrm{H}$-SiC since screw dislocations in $6 H$-SiC do not wind but almost all follow a straight or archlike (with a large curvature radius) trajectory. ${ }^{14}$ Moreover, apparently almost half of the screw dislocations have bimodal images, while the rest have unimodal images, and some of dislocations lying on the (0001) plane have more widely bimodal images. Because these differences in the dislocation images of the screw dislocations were not observed in Fig. 2(c) by synchrotron monochromatic x-ray topography, ${ }^{9}$ it is confirmed that the bimodal images are not due to pairs of two unimodal images of screw dislocations.

In the section topograph of Fig. 2(b), fringe-shaped images can be observed on the strip. The lateral positions of the fringe-shaped images exactly correspond to the positions of intersection points between the screw dislocations and the white broken line in Fig. 2(a). The fringe-shaped images are, therefore, due to the screw dislocations. Figure 3 shows a magnified image of a fringe-shaped image in Fig. 2(b). It is clear in this image that upper and lower fringes deviate slightly from each other in their lateral positions, and that the fringe-shaped images have tails on their outer edges. The shape profile of this image can accurately be described as hyperbolic. This hyperbolic profile of the section topograph indicates that the sense of the Burgers vector component parallel to the $c$ axis of this screw dislocation is right handed.

In the same way, we determined the senses of 32 screw dislocations observed on the section topograph in the range of $14.5 \mathrm{~mm}$ in width, as shown in Fig. 4. The image in Fig. 4 also reveals that the screw dislocations are inhomoge-

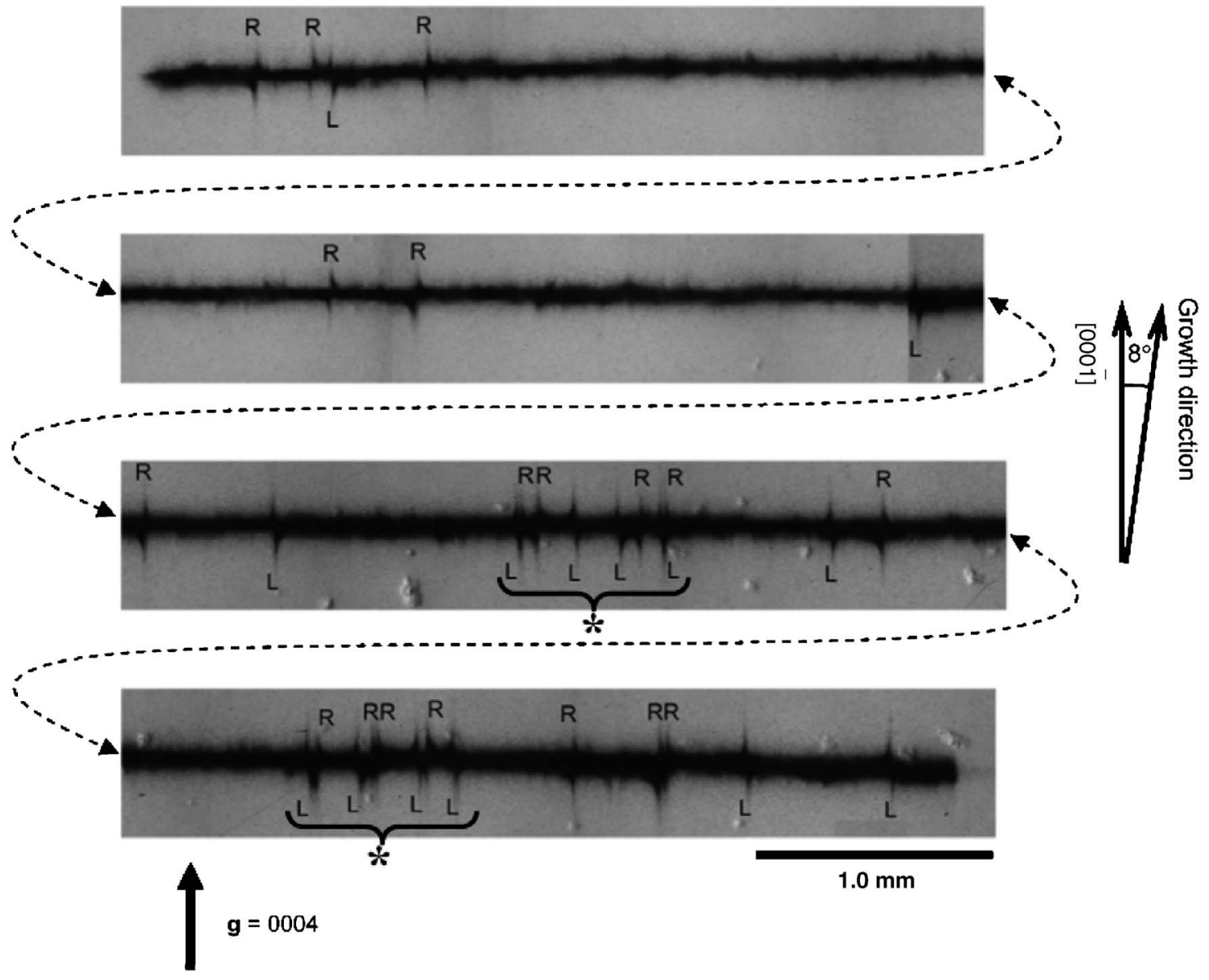

FIG. 4. Section topograph of $14.5 \mathrm{~mm}$ in width. Right-handed and left-handed dislocations are marked with $\mathbf{R}$ and $\mathbf{L}$, respectively. Regions with closely spaced screw dislocations are denoted by asterisk. Broken arrows indicate connections of the original section topograph. 
neously distributed, and there are regions (marked with the symbol of asterisk) with closely spaced screw dislocations. In those regions marked with asterisk, there exist exactly the same numbers of left-handed (marked with $\mathbf{L}$ ) and righthanded (marked with $\mathbf{R}$ ) screw dislocations.

In the whole regions covered by Fig. 4, there exist 14 left-handed and 18 right-handed screw dislocations. From a crystallographic viewpoint, there should be no difference between left-handed and right-handed dislocations in abundance ratio, as long as the crystal growth is not influenced by any external bias, e.g., inhomogeneous seed quality, inhomogeneous external stresses, and so on. Assuming that there is no difference in abundance ratio between the left-handed and right-handed screw dislocations, the population parameter is 32 , and normal distribution is appropriate to approximate, the expected number and standard deviation of each screw dislocation will be 16 and $\sim 3$, respectively. Accordingly, the abundance ratio experimentally obtained in the present study is within the value of standard deviation. We can conclude, therefore, that there is no significant difference in the abundance ratio between the left-handed and right-handed screw dislocations in the present crystal.

\section{DISCUSSION}

\section{A. Section topography}

In the previous studies, the magnitude of the Burgers vector of micropipe defects (with a hollow core) in hexagonal $\mathrm{SiC}$ single crystals has been revealed to be equal to or larger than $2 c(\sim 30 \AA)$ for $6 H-\mathrm{SiC}$ and equal to or larger than $3 c-4 c(30-40 \AA)$ for $4 H-S i C .{ }^{20-22}$ In other words, the screw dislocations (without a hollow core) in $4 H$-SiC crystals can have some types of the magnitudes of the Burgers vector component along the $c$ axis, that is, $1 c, 2 c$, or $3 c$. In this study, we determine which magnitude of the Burgers vector of screw dislocations is dominant in the RAF-grown $4 H$-SiC.

The optical geometry of the section topography employed here is very similar to that used by Si et al. to determine the Burgers vector magnitude of micropipe defects in $6 \mathrm{H}$-SiC single crystals. ${ }^{14}$ Owing to very small size of light source of the synchrotron x-ray white beam obtained in the SPring-8, it has been possible to make a specimen-to-film distance $D_{\text {sf }}$ larger (without resolution loss) by a factor of 10 when compared to that of previous works. We have been able, therefore, to directly determine the smaller magnitude $(\sim 1 \mathrm{~nm})$ of the Burgers vectors by means of the section topography with this long specimen-to-film distance $(2 \mathrm{~m})$. Assuming that the incident synchrotron white beam is strictly parallel, the profile of the displacement $Y$ recorded on the section topograph near screw dislocations can be simply expressed by the following formula:

$$
Y=b D_{\text {sf }} / \pi r,
$$

where $b$ is the magnitude of the Burgers vector and $r$ is the lateral distance from a dislocation core. ${ }^{14,23}$

According to this equation, theoretical hyperbolic curves for $b=1 c=10.05 \AA, 2 c=20.11 \AA, 3 c=30.16 \AA$, and $4 c$ $=40.21 \AA$, shown in Fig. 3, are drawn on the section topo- graph around a screw dislocation. It is obvious in Fig. 3 that the theoretical curve for $b=1 c$ best fits the experimental profile. This indicates that the screw dislocation in Fig. 3 has $1 c$ in its Burgers vector component. Moreover, we confirmed that all the other screw dislocations (about 31 dislocations) observed here also have $1 c$ in their Burgers vector components. Thus, we can conclude that screw dislocations with $1 c$ in their Burgers vector components are generally dominant in RAF-grown $4 H$-SiC single crystals.

The fact that the numbers of left-handed and righthanded screw dislocations are the same in the regions denoted by asterisk in Fig. 4 suggests that the screw dislocations might tend to have opposite senses to adjacent screw dislocations. Actually, the number of cases that two adjacent screw dislocations in Fig. 4 have opposite senses (in the case of L-R or R-L) is 21, and that of the same senses is 10 (in the case of L-L or R-R); that is, two adjacent screw dislocations have statistically significant tendency toward opposite senses. The authors speculate that there could be two kinds of potential causes that would explain this tendency. One is that the source of the screw dislocations is the generation of dislocation half loop due to stress concentrator (carbon precipitation, Si droplet, etc.), and the pair of screw dislocations with opposite senses propagates along the direction of growth (almost without changing the direction of propagation during the bulk growth). The second potential cause is the possibility that the elastic interaction between screw dislocations affects the direction of propagation (opposite and same senses resulting in attractive and repulsive elastic forces, respectively) resulting in both gathered and scattered screw dislocations. As we mentioned concerning the line shape of screw dislocations shown in Fig. 2(a), screw dislocations can change their propagating direction in a winding fashion, even perpendicular to the growth direction. This fact suggests that the first potential cause cited above is not sufficient to thoroughly explain this phenomenon. Accordingly, the second cause, the elastic interaction between adjacent screw dislocations, must play an important role in the manner of propagation and distribution of screw dislocations.

To approximately estimate the range of the elastic interaction between screw dislocations, we plotted the distribution of spacing between two adjacent screw dislocations, as shown in Figs. 5(a) and 5(b), for adjacent dislocations with opposite and same senses, respectively. Because the values for spacing between two adjacent screw dislocations in Fig. 5 were directly measured from the section topograph of Fig. 4 (ignoring the spacing along the thickness direction), the spacing values for some of two adjacent screw dislocations might be underestimated, especially in the small spacing region $(<400 \mu \mathrm{m})$. This plot, however, shows a good tendency of the spacing distribution. It is clear from Fig. 5 that the two adjacent screw dislocations with opposite senses [Fig. 5(a)] strongly tend to have small spacing under $200 \mu \mathrm{m}$ (or even under $100 \mu \mathrm{m}$ ), while the two adjacent screw dislocations with the same senses [Fig. 5(b)] do not show clear tendency in spacing. The result of Fig. 5(a), at least, should be attributed to the attractive interaction between the two adjacent screw dislocations with opposite senses. Based on these results, we propose that the effective range (effective 
(a)

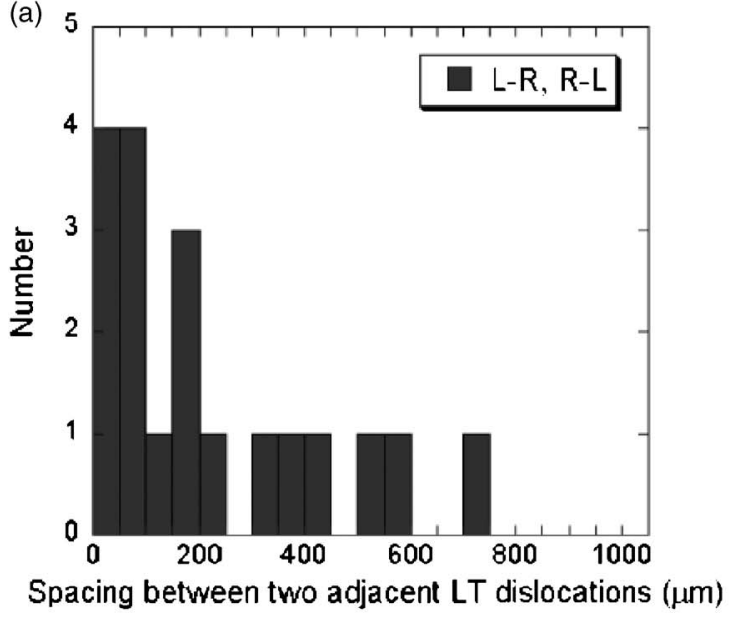

(b)

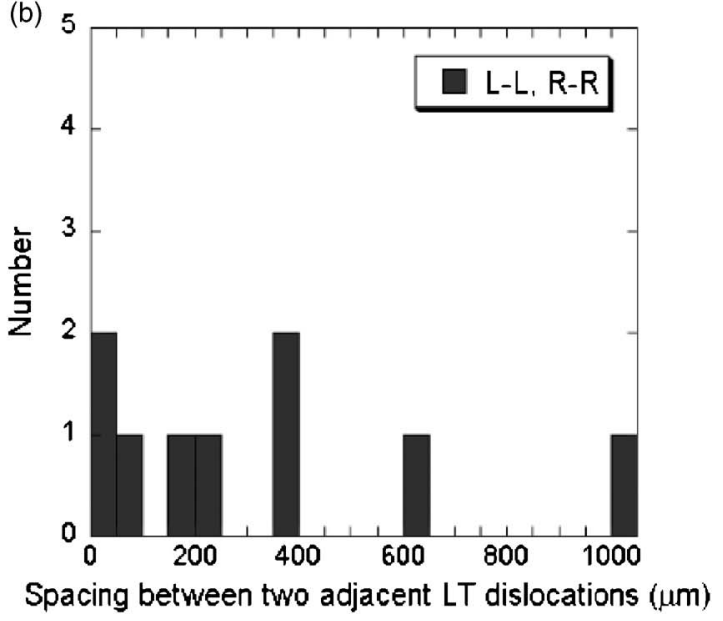

FIG. 5. Histograms for the spacing between two adjacent screw dislocations with opposite senses (a) and with the same senses (b). It can be recognized that two adjacent screw dislocations with the opposite senses strongly tend to have small spacing under $200 \mu \mathrm{m}$, while the two adjacent screw dislocations with the same senses.

enough to affect the dislocation-propagation process) of elastic interaction between screw dislocations during crystal growth is about $200 \mu \mathrm{m}$ or more.

Polarized light microscopy (PLM) analysis, on the other hand, has revealed that the range of stress field around elementary screw dislocations are about a few tens of microns at room temperature ${ }^{22,24}$ and that at growth temperature $\left(>2000{ }^{\circ} \mathrm{C}\right)$ is supposed to be much smaller than that at room temperature. ${ }^{25}$ In comparison, our proposed effective range of $200 \mu \mathrm{m}$ or more is much larger than that which has been supposed through PLM analysis. We think that this discrepancy must be attributed to the stress-sensitivity limit of the PLM. In a theoretical sense, the range of stress field around a dislocation is infinite, and the elastic force between the two dislocations is inversely proportional to their spacing. ${ }^{26}$ Therefore, there must be very weak elastic interaction between far apart screw dislocations even over the range of stress field observed through PLM. We believe that this weak and long-range elastic interaction changes the direction of dislocation propagation, making the screw dislocations gather, and resulting in inhomogeneous dislocation distribution. This is the first concrete report that weak and long-range elastic interactions between elementary dislocations play an important role in governing the behavior of dislocation propagation during crystal growth.

\section{B. Projection topography}

As mentioned above, almost half of the dislocation images in the white x-ray projection topograph [Fig. 2(a)] have bimodal profiles, while the rest of them have unimodal profiles. To clarify the relationship between the dislocation sense and the dislocation-image profile, we have reproduced a dislocation image, as shown in Fig. 2(d), in which the righthanded and left-handed dislocations are indicated by black and white, respectively. It is clear at a glance that the righthanded and left-handed dislocations tend to have unimodal and bimodal profiles in their images. Strictly speaking, the left-handed dislocations tilt clockwise and the right-handed

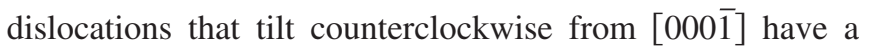

bimodal and relatively wide profile, while the left-handed dislocations tilted counterclockwise and the right-handed dislocations clockwise have a unimodal and relatively narrow profile. This image illustrates a clear relationship between dislocation sense, dislocation-image width, and

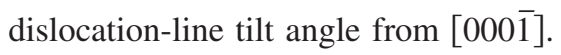

To evaluate this relationship in detail, we plotted the dislocation-image width observed at several points of ten screw dislocations with respect to dislocation-line tilt angle from [0001] , as shown in Fig. 6(a) It is obvious that the image width of the left-handed dislocation (marked with open and filled squares) increases as the dislocation-line tilt angle increases clockwise, and that the image width of the right-handed dislocation (marked with open and filled circles) decreases and reaches a saturation point as the dislocation-line tilt angle increases clockwise. Further, in terms of crystallographic equivalence, the curves for righthanded and left-handed dislocations are thought to have mirror symmetry with respect to vertical axis at an angle of $0^{\circ}$. At a tilt angle of $90^{\circ}$, which corresponds to basal-plane dislocations with a pure edge character, their image widths can be explained in terms of beam divergence and convergence due to effective distortion around edge dislocation, ${ }^{27}$ as shown in Fig. 6(b). The x-ray beam diffracted at the lobe of distorted lattice is diverged or converged, depending on the sense, resulting in a wide and bimodal profile or a narrow and unimodal profile on the film. This discussion must be expanded to a mixed dislocation since it possesses both screw and edge characters. Those dislocations tilted at about $1^{\circ}-30^{\circ}$ in the present crystal are very mixed dislocations and have a considerable edge character (which increases as tilt angle increases).

To confirm this discussion, we carried out ray-tracking simulation to obtain the tendency of the image width of dislocations with the mixed characters. The geometry for the simulation is shown in the inset of Fig. 6(a). The mixed dislocation with the Burgers vector of $b=1 c$ and with the tilt angle $\theta$ (between dislocation line and [0001]) can be interpreted as a superposition of pure screw and pure edge dislo- 


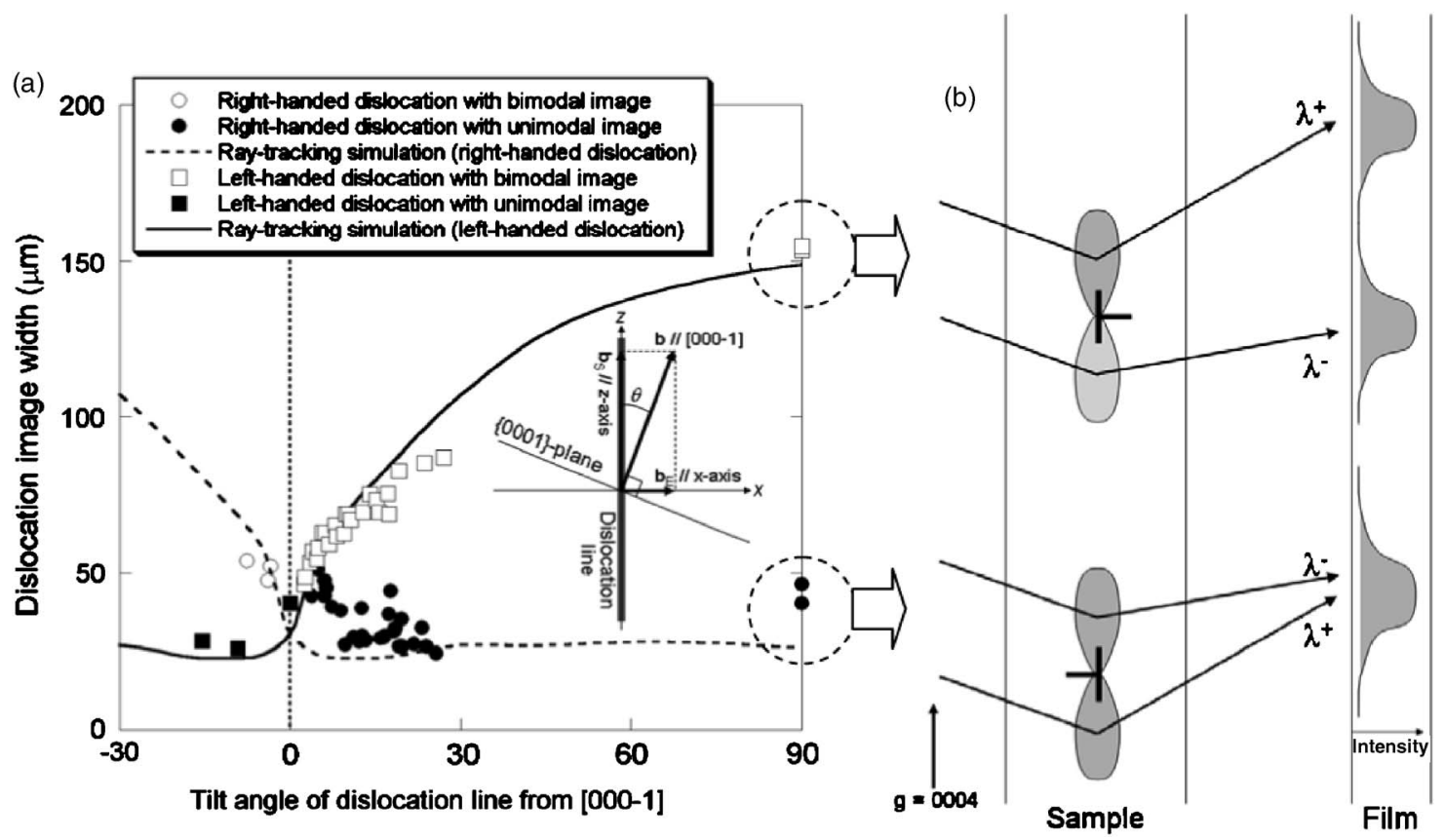

FIG. 6. Dislocation-image width dependences on tilt angle of dislocation line from [0001] (a). The inset in (a) is a geometry for a ray-tracking simulation. Schematic illustrations of bimodal and unimodal image formation mechanism at the tilt angle of $90^{\circ}$ are drawn on the top and bottom of (b), respectively.

cations with the Burgers vectors of $b_{S}=b \cos \theta$ and $b_{E}$ $=b \sin \theta$, respectively. The lattce displacements around the dislocation in Cartesian coordinates are expressed as follows: ${ }^{26}$

$$
u_{x}=\frac{b_{E}}{2 \pi} \arctan \left(\frac{y}{x}\right), \quad u_{y}=0, \quad u_{z}=\frac{b_{S}}{2 \pi} \arctan \left(\frac{y}{x}\right) .
$$

Differentiating them with respect to the spatial coordinates gives the normal vector of the local $\{0001\}$ plane. Then, the diffracted $\mathrm{x}$-ray intensity profile on the film can be obtained by the ray-tracking simulation using the local dependency of the normal vector. ${ }^{16}$ The criterion to determine dislocation width was chosen to $15 \%$ foot with respect to the peak of "effective intensity profile" (which is obtained by subtracting the matrix intensity from the simulated x-ray intensity profile). The simulated dislocation-width dependences with respect to dislocation-line tilt are shown in Fig. 6(a). The results for right-handed and left-handed dislocations are denoted as the broken and solid lines, respectively. It is clear that the simulated results fit the experimental results very well. Thus, we conclude that the edge character due to dislocation-line tilt makes the dislocation-image bimodal (wide) or unimodal (narrow) depending on the sense of dislocation.

It is very noteworthy to mention that, in fact, this simple white $\mathrm{x}$-ray projection topography with an extremely long sample-to-film distance can determine the Burgers vector sense of dislocations (except a pure screw dislocation) by measuring only dislocation-image width and dislocation-line tilt angle. Taking into consideration the fact that the Burgers vector magnitude can also be determined through the comparative analysis of white $\mathrm{x}$-ray projection topography, ${ }^{14,15}$ this simple technique (employing third-generation synchrotron facility) can be a powerful tool to effectively examine a detailed dislocation structure (including information on the Burgers-vector direction, sense, and magnitude of dislocations) in any single crystals.

\section{CONCLUSION}

The authors have investigated the elementary screw dislocations in a high-quality $\mathrm{SiC}$ single crystal using synchrotron white $\mathrm{x}$-ray topography. We have determined the senses and magnitudes of their Burgers vector using section topography with a long sample-to-film distance, thanks to a highly coherent beam in a third-generation synchrotron facility. We have demonstrated that the distribution of the dislocations are significantly related to the sense of the dislocations, which is thought to be due to weak and long-range $(>200 \mu \mathrm{m})$ elastic attractive and/or repulsive interactions between dislocations with opposite senses and/or same senses. To the authors' knowledge, this is the first report that such a long-range interaction between elementary dislocations can affect the propagation behavior and distribution of the dislocations. Moreover, we have found that a simple projection topography employing third-generation synchrotron facility with an extremely long sample-to-film distance can also determine the sense of elementary dislocations with edge characters. This technique could be very useful in examining detailed dislocation structure and their physics, especially in developing compound semiconductor crystals (not only $\mathrm{SiC}$ but also nitrides, oxides, etc.) and their devices which still contain a large density of dislocations to be potentially harmful both to device performance and reliability.

\section{ACKNOWLEDGMENTS}

The authors would like to thank the research team of Denso Corporation for their dedicated help. The synchrotron 
white $\mathrm{X}$-ray topography experiments were performed at the BL28B2 in the SPring-8 with the approval of the Japan Synchrotron Radiation Research Institute (JASRI) (Proposal No. 2006B0176).

${ }^{1}$ M. Bhatnagar and B. J. Baliga, IEEE Trans. Electron Devices 40, 645 (1993).

${ }^{2}$ H. Matsunami and T. Kimoto, Mater. Sci. Eng., R. 20, 125 (1997).

${ }^{3}$ H. Morkoc, S. Strite, G. B. Gao, M. E. Lin, B. Sverdlov, and M. Burns, J. Appl. Phys. 76, 1363 (1994).

${ }^{4}$ J. J. Sumakeris, J. R. Jenny, and A. R. Powell, MRS Bull. 30, 280 (2005).

${ }^{5}$ St. G. Müller, M. F. Brady, A. A. Burk, H. Mcd. Hobgood, J. R. Jenny, R.

T. Leonard, D. P. Malta, A. R. Powell, J. J. Sumakeris, V. F. Tsvetkov, and

C. H. Carter, Superlattices Microstruct. 40, 195 (2006).

${ }^{6}$ H. Lendenmann, F. Dahlquist, N. Johansson, R. Soderholm, P. A. Nilsson, J. P. Bergman, and P. Skytt, Mater. Sci. Forum 353-356, 727 (2000).

${ }^{7}$ P. G. Neudeck, W. Huang, and M. Dudley, IEEE Trans. Electron Devices 46, 478 (1999).

${ }^{8}$ D. Nakamura, I. Gunjishima, S. Yamaguchi, T. Ito, A. Okamoto, H. Kondo, S. Onda, and K. Takatori, Nature (London) 430, 1009 (2004).

${ }^{9}$ D. Nakamura, S. Yamaguchi, I. Gunjishima, Y. Hirose, and T. Kimoto, J. Cryst. Growth 304, 57 (2007).

${ }^{10}$ A. O. Okunev, L. N. Danil'chuk, and V. A. Tkal', Phys. Solid State 48, 2084 (2006).

${ }^{11}$ Y. Chen, G. Dhanaraj, M. Dudley, E. K. Sanchez, and M. F. MacMillan, Appl. Phys. Lett. 91, 071917 (2007).
${ }^{12}$ Y. Chen and M. Dudley, Appl. Phys. Lett. 91, 141918 (2007).

${ }^{13}$ Y. M. Tairov and V. F. Tsvectov, J. Cryst. Growth 43, 209 (1978).

${ }^{14}$ W. Si, M. Dudley, R. Glass, V. Tsvektov, and C. Carter, J. Electron. Mater. 26, 128 (1997)

${ }^{15}$ W. Si, M. Dudley, R. Glass, V. Tsvektov, and C. Carter, Mater. Sci. Forum 264-268, 429 (1998).

${ }^{16}$ X. R. Huang, M. Dudley, W. M. Vetter, W. Huang, S. Wang, and C. H. Carter, Appl. Phys. Lett. 74, 353 (1999).

${ }^{17}$ J. Härtwig, J. Baruchel, H. Kuhn, X. R. Huang, M. Dudley, and E. Pernot, Nucl. Instrum. Methods Phys. Res. B 200, 323 (2003).

${ }^{18}$ S. Ha, M. Skowronski, W. Vetter, and M. Dudley, J. Appl. Phys. 92, 778 (2002).

${ }^{19}$ Q. Wahab, A. Ellison, A. Henry, E. Janzén, C. Hallin, J. Di Persio, and R. Martinez, Appl. Phys. Lett. 76, 2725 (2000).

${ }^{20}$ N. Sugiyama, A. Okamoto, K. Okumura, T. Tani, and N. Kamiya, J. Cryst. Growth 191, 84 (1998).

${ }^{21}$ M. Dudley, X. R. Huang, and W. Vetter, J. Phys. D 36, A30 (2003).

${ }^{22}$ X. Y. Ma, J. Appl. Phys. 99, 063513 (2006).

${ }^{23}$ S. Mardix, A. R. Lang, and I. Blech, Philos. Mag. 24, 683 (1971).

${ }^{24}$ X. Y. Ma, M. Dudley, W. Vetter, and T. Sudarshan, Jpn. J. Appl. Phys., Part 2 42, L1077 (2003)

${ }^{25}$ T. Kato, "Crystallographic study on substrates for $\mathrm{GaN}$ film and $\mathrm{GaN}$ epitaxial growth," Ph.D. thesis (Nagoya Institute of Technology, 1998).

${ }^{26}$ D. Hull and D. J. Bacon, Introduction to Dislocations, 4th ed. (ElsevierButterworth-Heinemann, Oxford, 2001), Chap. 4.

${ }^{27}$ D. K. Bowen and B. K. Tanner, High Resolution X-ray Diffractometry and Topography (Taylor \& Francis, London, 2001), Chap. 10. 